\title{
Contralateral prophylactic mastectomy and implications for breast reconstruction
}

\author{
Katherine B. Santosa ${ }^{1}$, Jeremie D. Oliver ${ }^{2}$, Adeyiza O. Momoh ${ }^{1}$ \\ ${ }^{1}$ Section of Plastic Surgery, Department of Surgery, University of Michigan, Ann Arbor, MI, USA; ${ }^{2}$ Department of Biomedical Engineering, School \\ of Dentistry, School of Medicine, University of Utah, Salt Lake City, UT, USA \\ Contributions: (I) Conception and design: KB Santosa, AO Momoh; (II) Administrative support: AO Momoh; (III) Provision of study materials or \\ patients: None; (IV) Collection and assembly of data: All authors; (V) Data analysis and interpretation: All authors; (VI) Manuscript writing: All \\ authors; (VII) Final approval of manuscript: All authors. \\ Correspondence to: Adeyiza O. Momoh, MD. Associate Professor, Section of Plastic Surgery, Department of Surgery, Michigan Medicine, 1500 E. \\ Medical Center Drive, Ann Arbor, MI 48109, USA. Email: amomoh@med.umich.edu.
}

\begin{abstract}
Contralateral prophylactic mastectomy (CPM) rates have continued to rise in the United States, impacting all stakeholders including plastic and reconstructive surgeons. Multiple factors may be influencing this trend, including patient decision-making characteristics, knowledge about breast cancer disease and prognosis, advances in genetic testing and enhanced imaging capabilities, sociodemographic factors, and access to specialty surgical services such as breast reconstruction. In this review, the authors shed light on the current state of CPM and summarize the literature analyzing its increasing prevalence in the United States, as well as outline future directions for study and dissemination of knowledge from providers to patients surrounding this important and complex treatment decision.
\end{abstract}

Keywords: Prophylactic mastectomy; patient outcome assessment; postoperative complications

Submitted Jan 30, 2020. Accepted for publication Feb 15, 2020.

doi: 10.21037 /gs.2020.03.15

View this article at: http://dx.doi.org/10.21037/gs.2020.03.15

\section{Introduction}

Despite a notable decline in the incidence of contralateral breast cancer in the United States, women are choosing to undergo contralateral prophylactic mastectomy (CPM) for earlier-stage, lower-risk unilateral breast cancer at an increasing rate (1). Although there is no significant survival benefit for low to average risk patients who undergo CPM, there has been an estimated increase in CPM rates in the United States of $139 \%$ since 2004 (1). Furthermore, the proportion of breast conserving procedures performed for treatment of early stage breast cancer has declined, with a compensatory rise in the number of patients opting for CPM (2).

In women with early stage breast cancer who have undergone a unilateral mastectomy, the risk for contralateral breast cancer has been reported to be 4 percent over 10 years (3). Large population-level studies have demonstrated that CPM does not offer an improvement in either breast cancer-specific survival or overall survival among women with unilateral stage I to III breast cancer (4). Additionally, the likelihood of finding an occult breast cancer in the CPM specimen that was not detected on imaging is also low, estimated to occur 1 to 2 percent of the time $(5,6)$. Although there have not been prospective randomized studies demonstrating a survival benefit in CPM, there is some evidence to suggest an approximate reduction in risk of cancer in the contralateral breast by 95 percent (7). Along these lines a few studies have shown evidence of some survival benefit in younger women ( $<49$ years) and in women with estrogen receptor-negative disease (8-10). An updated Cochrane Review of 61 studies suggests that while CPM reduces the incidence of contralateral breast cancer, there is insufficient evidence that CPM improves survival among patents and that selection bias in healthier, younger women opting for CPM may produce overall survival for 
these patients (11).

With shifts towards more aggressive oncologic surgical treatment, breast reconstruction rates and trends have been impacted as patients who undergo bilateral mastectomies have been shown to be more likely to pursue breast reconstruction when compared to similar patients undergoing unilateral mastectomies (12). Attempts to decipher factors influencing this trend toward increasing CPM rates and how they relate to breast reconstruction have suggested that it seems to be patient-driven ranging from fear of recurrence and anxiety to desire for symmetry (13-15).

This review article seeks to provide an overview of CPM and its implications on breast reconstruction with a focus on decisional factors, potential complications, quality of life (QOL) implications and the cost to society as a whole. As we gain a better understanding of these issues, it is crucial that we all engage with patients on the existing evidence to facilitate well informed shared decisions for oncologic and reconstructive management.

\section{Factors influencing choice to undergo prophylactic contralateral mastectomy}

Decision making for breast cancer treatment can be complicated with newly diagnosed patients attempting to understand and navigate various surgical options available to them (9). The ramifications of these decisions on survival and life after treatment are important to consider. Here, we discuss specific factors that have been found to be influential in decisions made regarding CPM.

\section{Advances in genetic testing, imaging and oncologic management}

Key advances in personalized cancer therapy in modern medicine have been made, including the development and availability of genetic testing for breast cancer-specific gene mutations and the increased use of high-definition medical imaging such as magnetic resonance imaging (MRI) and three-dimensional image software for cancer detection purposes. Women with the BRCA1/2 mutation for instance, have an increased lifetime risk between 56$87 \%$ of developing breast cancer, as well as an increased likelihood of developing a second contralateral or ipsilateral primary breast cancer, lending support to the importance of precision medicine initiatives to deliver personalized curative therapy for patients with specific genetic mutations predisposing to pathology $(16,17)$. Several studies have demonstrated the distinct benefit CPM can have to mitigate the risk of future breast disease burden by 90-97\% among patients with BRCA mutation $(16,17)$. Of note, about $50 \%$ of patients with confirmed BRCA mutation in the US opt to undergo mastectomy to treat the primary cancer and CPM to excise the uninvolved breast tissue (17). Given the well-described potential benefit for oncologic risk reduction of CPM in such patients, the NCCN guidelines and Preventive Service Task Force recommends that CPM should be a critical area of discussion for all patients who are diagnosed with the BRCA mutation (18).

Additionally, the increased availability of MRI to patients with newly diagnosed breast cancer strongly contributes to the receipt of CPM (19-23). Breast MRI is used as an additional tool to visualize mammographically occult breast lesions and is more sensitive than mammography in detecting occult breast lesions in either the affected and/or unaffected contralateral breast $(24,25)$. Proponents for its use argue that it can lead to a reduction in re-excision surgery and local recurrences (26). However, more recent data suggests that MRI should not be routinely used before oncologic surgery as it can create false positives and does not reduce re-excision rates (26). Interestingly, MRI before oncologic breast surgery impacts surgical decision in favor of mastectomy over breast conserving therapy, and CPM $(20,26)$. While breast MRI is a recommendation by the American Cancer Society as a part of the surveillance for women with confirmed BRCA mutation, when the impact of MRI on mastectomy choice was studied, a positive association between the two was observed (19). Firstly, Katipamula and colleagues (20) identified a 50\% increased use of breast MRI with simultaneous rise of CPM at their institution; subsequently, Miller and colleagues (21) and Sorbero and colleagues (22) also reported increased prevalence of women opting for CPM after receiving breast MRI for preoperative surgical planning for oncologic breast surgery.

\section{Decisional characteristics}

The emotional toll of a breast cancer diagnosis and therapeutic journey each patient is faced with continues to be a key driver toward increasing rates of CPM and is a crucial factor for which clinicians need to be acutely aware when discussing therapeutic options with patients and their families. Evidence strongly suggests that utilization of CPM is largely driven by patient anxiety about the potential for experiencing a subsequent breast cancer diagnosis and a 


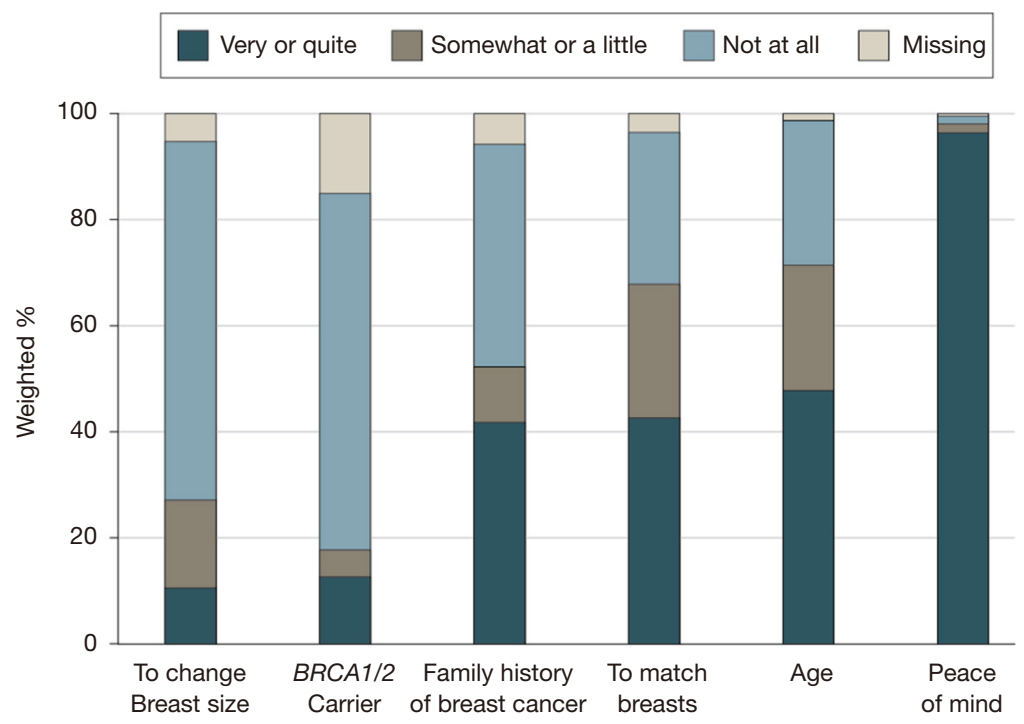

Figure 1 Factors driving patient decision-making toward contralateral prophylactic mastectomy (used with permission from Jagsi R, Hawley ST, Griffith KA, et al. Contralateral Prophylactic Mastectomy Decisions in a Population-Based Sample of Patients With Early-Stage Breast Cancer. JAMA Surg 2017;152:274-82).

desire for peace of mind (Figure 1) (13-15,27). In a recent multi-institutional study of young women who underwent CPM, the most highly ranked motivating factor driving the decision to undergo CPM was a desire to minimize risk of a second breast cancer, and nearly all patients surveyed stated that this was a very important factor in their decision to ultimately undergo CPM (28).

Studies have shown that levels of anxiety and worry about cancer may actually be higher before surgery among women who opt for CPM compared to those who do not $(29,30)$. Moreover, CPM may have positive effects on psychosocial well-being, as anxiety and worry among women who have CPM decline to levels similar to women who did not choose CPM (30). Additionally, among women with unilateral breast cancer and a family history of breast cancer who were surveyed 20 years after undergoing CPM, 90\% were satisfied or very satisfied with their decision to undergo the procedure (31), further emphasizing the potential benefits of CPM on patient satisfaction and emotional well-being.

Knowledge about breast cancer also impacts decisions made to pursue CPM or unilateral mastectomy. Women who are less knowledgeable about breast cancer are more likely to be interested in and choose CPM $(27,29)$ perhaps because they are unaware of the increased risk in complications and/or the relatively low risk of developing a contralateral breast cancer and the lack of clear benefit in survival. Additionally, when surgeons educate and recommend against CPM in patients without clinical indications, utilization of CPM is low (15). This highlights the importance of engagement by providers in discussions with women faced with these decisions to provide comprehensive information and to actively engage in shared decision-making.

\section{Sociodemographic determinants}

Adding to the complexity of CPM are the effects of sociodemographic factors on patient decisions. Several studies have demonstrated that race is significantly associated with surgical decision-making, with White patients being more likely than non-White patients to undergo CPM $(2,15,19,32)$. Although few studies have examined reasons for the disparity, $\mathrm{Yi}_{1}$ and colleagues suggest that the difference in CPM utilization between races may be due to differences in perceived risks and perceived efficacy of CPM (32). These authors found that Black women reported lower perceived risks of a contralateral breast cancer and viewed CPM as less effective in lowering that risk than White women in their study (32). These findings are supported by other studies that have shown that White women in general are more likely to overestimate the risk of developing breast cancer than minority women $(33,34)$.

Additionally, there is substantial evidence to suggest that CPM may be positively associated with increased 
socioeconomic status and higher education level $(14,15,35)$. Reasons for this are unclear but could be related to patients of higher socioeconomic status taking greater control in deciding on treatment options for their breast cancer as opposed to relying on recommendations made by providers. A qualitative study on the decision-making process for CPM amongst women treated at a single academic center found that $88 \%$ of women in the study population underwent CPM based on patient preference as opposed to physician recommendation. Over a quarter of women in the study (29\%) admitted to making up their minds for CPM prior to the consultation (13). Whatever reasons are driving these health disparities in CPM, it is important to note that racial minorities, patients from lower socioeconomic classes, and patients with less education encounter more barriers to healthcare, and experience different, often inferior, communication with their physicians (32,36-38).

Disparities exist in the receipt of breast reconstruction among all patients—not just those who are considering CPM. Importantly, sociodemographic factors such as race, use of English as a second language, and lower income and insurance status have been found to be associated with a lower chance of having a conversation about breast reconstruction with a physician or going onto receive postmastectomy breast reconstruction (39). As such, there must be efforts from the healthcare system and individual providers to standardize the discussion surrounding $\mathrm{CPM}$ and of breast reconstruction overall to each patient, regardless of racial, economic or educational background to ensure that these disparities do not limit access or adversely affect patient outcomes.

\section{Surgeon and center characteristics}

Surgeon and center attributes may also impact the receipt of CPM. With regards to the individual surgeon, Katz and colleagues found that the attending breast surgeon explained $20 \%$ of the variability in the receipt of CPM after controlling for patient factors. In addition, women in their analysis were nearly three-times as likely to receive CPM if their breast surgeon tended to perform more CPM than their peers (40), highlighting the impact of an individual surgeon on the decision-making process. Moreover, center attributes such as access to immediate breast reconstruction has been shown to significantly impact utilization of CPM (41), and that discussions with plastic surgeons may drive patients towards bilateral versus unilateral surgery $(41,42)$. In a qualitative study by Covelli, they suggest that the higher utilization of CPM in the US compared to Canada may be attributable to the notion that US surgeons discuss reconstructive options early in the consultation process per legislative guidelines, but that Canadian surgeons only discuss reconstruction when patients were considering mastectomy (41).

However, new data from a survey of plastic surgeons in two large metropolitan areas of the United States, suggests that patients (not plastic surgeons) initiate most (82.8\%) of the discussions regarding CPM during consultations with women with unilateral breast cancer and no increased risk for contralateral breast cancer. Moreover, the study found that plastic surgeons although not driving the conversation, do not generally discourage patients from considering CPM even if it is not medically indicated given their low risk for contralateral breast disease (43). These findings provide important insights suggesting that although consultations with plastic surgeons may be a factor in CPM utilization as previous studies have shown, that it is unlikely that plastic surgeons are encouraging patients to undergo bilateral breast surgery when it would otherwise not be medically indicated. This also suggests that plastic surgeons are not discouraging CPM, but are honoring and respecting their patients' decisions in pursuing CPM and bilateral breast reconstruction. What remains clear is that plastic and reconstructive surgeons can play pivotal roles in the shared decision-making process with patients and should be wellinformed about the issues surrounding CPM.

\section{Breast reconstruction and CPM}

A significant factor in an individual patient's desire to pursue CPM and affecting the overall higher utilization rates of CPM on a population level is breast reconstruction $(19,32)$. There are several considerations that are important to highlight when addressing the topic of breast reconstruction and CPM such as complication data, desire for symmetry and improved aesthetics, and patient-reported outcomes such as satisfaction and breast-related QOL.

\section{Morbidity associated with CPM and breast reconstruction}

Major downsides to CPM are the potential for higher complication rates associated with bilateral surgery and a longer recovery time $(35,44-46)$. A single-center, retrospective study by Miller and colleagues demonstrated that patients undergoing CPM were 2.7 times more likely to be readmitted or require additional interventions than 


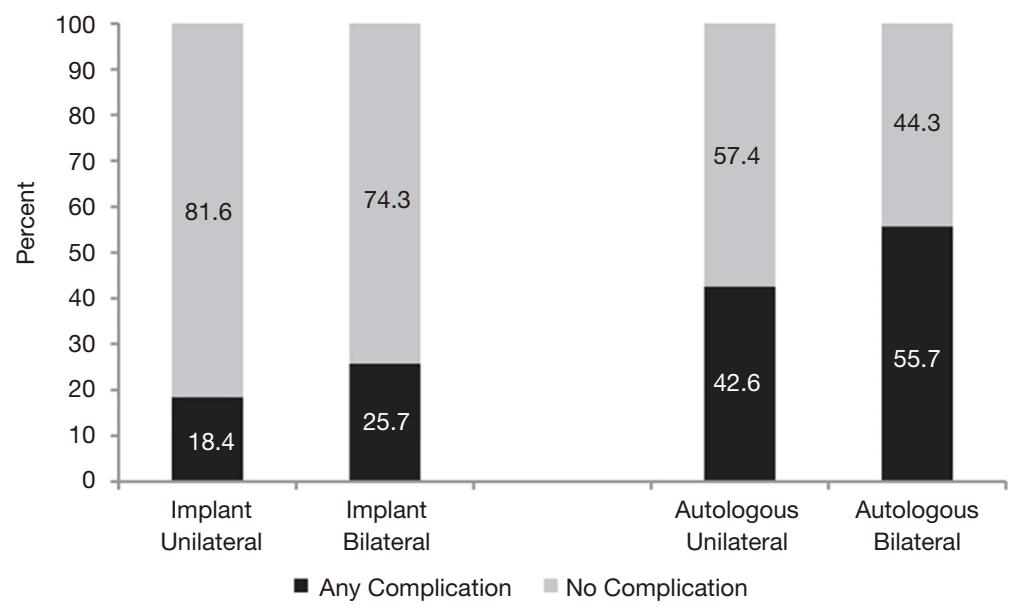

Figure 2 Postoperative complication rate following unilateral and bilateral implant-based and autologous breast reconstruction (used with permission from Momoh AO, Cohen WA, Kidwell KM, et al. Tradeoffs Associated With Contralateral Prophylactic Mastectomy in Women Choosing Breast Reconstruction: Results of a Prospective Multicenter Cohort. Ann Surg 2017;266:158-64).

those who did not (46). In a large multicenter, prospective study evaluating outcomes of women with unilateral breast cancer who underwent unilateral versus bilateral mastectomy with reconstruction, Momoh and colleagues found that regardless of reconstruction type (i.e., implant-based versus autologous) that women with bilateral reconstructions had significantly greater complication rates than women who opted for unilateral reconstruction (Figure 2). Additionally, after controlling for patient and demographic factors, their analysis also demonstrated that CPM was independently associated in the development of complications after surgery (35), which has also been corroborated by other studies (44-46).

Although higher complication rates are frequently cited as problematic with CPM $(15,23,35)$, a few studies have suggested otherwise $(44,47,48)$, which may be due to limitations in study design and follow-up. Recently, a single center, retrospective analysis showed no difference in complication risk between women who underwent unilateral or bilateral mastectomy with immediate breast reconstruction (48). As this study draws from experience at a single institution, it is possible that these results may not be generalizable to other patients treated at different centers across the US. Additionally, according to a NSQIP analysis of 30-day complications, there were no differences in surgical site infections, prostheses failure or medical complications between women who underwent unilateral or bilateral mastectomy with reconstruction (44). It is prudent to consider however, that 30-day follow-up may not be adequate to fully assess complications of breast reconstruction as late complications are not atypical $(49,50)$. Another population-level study by Billig and colleagues did not reveal a significant difference in complication rates at 30-days and 18 months after surgery between unilateral versus CPM, although the authors cite the difficulty of assessing complications after breast reconstruction using billing codes in administrative claims databases like the one that was used in their analysis (47).

\section{Patient reported outcomes}

Patient-centered data are arguably the most important metric when evaluating breast reconstruction outcomes as it can be a very personal and intimate process (51). Across all patients undergoing breast reconstruction for cancer and prophylactic reasons, bilateral reconstruction seems to offer an advantage in satisfaction with breast as compared to unilateral reconstruction, likely reflecting an ability to control for symmetry in bilateral reconstructions (51). Similarly, when specifically evaluating patient-reported outcomes after breast reconstruction between women who choose CPM and those who do not, there seems to be a positive effect of CPM on satisfaction as well (52), and an effect of type of reconstruction (i.e., implant-based versus autologous) (35).

After surveying thousands of volunteers recruited from the Army of Women undergoing all different types of reconstruction after unilateral and CPM, Hwang and colleagues demonstrated that CPM was associated with 
higher satisfaction with breasts but at the cost of lower physical well-being and psychosocial well-being than nonCPM patients (52). Importantly, reconstructive type was not controlled for in their analysis and seems to be important in patient-reported outcomes after CPM. Among patients with unilateral breast cancer, patients who underwent unilateral implant-based reconstruction were significantly less satisfied with their breasts compared to women who opted for CPM and bilateral reconstruction. However, in women undergoing autologous breast reconstruction, there was no difference in satisfaction or breast-related QOL between those with CPM versus those with unilateral mastectomy and reconstruction, emphasizing the impact of procedure type of these outcomes (35).

The negative impact of implant-based reconstruction on patient-reported outcomes after unilateral versus bilateral breast reconstruction is likely attributed to the difficulty in obtaining symmetry between a breast reconstructed with an implant and a native contralateral breast. These challenges in obtaining symmetry or accounting for ptosis of the native breast compared to the reconstructed breast may underlie the decisions for women to opt for CPM instead of procedures for symmetry such as a reduction or mastopexy of the contralateral native breast $(35,53)$.

Women who undergo unilateral mastectomy obtain superior aesthetic results with autologous versus implant-based techniques (53). Several studies have also demonstrated that patients who elect for autologous reconstructive techniques report higher levels of satisfaction with their breasts and improvements in breast-related QOL compared to their pre-operative baseline scores $(35,51)$. Taken together, experts argue that a possible solution for the perceived overutilization of CPM among patients with low-risk breast cancer may be to advocate for autologous reconstruction among patients undergoing unilateral mastectomy as this could minimize complications (by performing unilateral versus bilateral surgery) while leveraging the benefits of improved satisfaction and wellbeing seen with bilateral reconstructions (35). Importantly, patients faced with the decision of whether or not to pursue CPM and elect for reconstruction should be informed that reconstructive type matters. These nuanced discussions with women faced with the decision to pursue CPM or not emphasize the importance of plastic surgeons in patient education and active engagement in the shared decisionmaking process.

Despite added operative time, longer recovery and higher complication rates from bilateral versus unilateral breast surgery, women continue to opt for CPM with reconstruction. This likely reflects that women are willing to accept the potential tradeoffs for the perceived benefits of CPM including (but not limited to) improved satisfaction and QOL.

\section{Financial considerations of CPM}

In addition to the oncologic data lacking support for substantial benefit of CPM for patient disease-free survival, discrepancies regarding financial implications have argued against low-toaverage-risk women undergoing CPM. Some studies have demonstrated higher healthcare costs of CPM in averagerisk women in the short-term $(54,55)$; reasons for the increase in short-term costs include the higher likelihood of patients who undergo CPM to also undergo bilateral reconstruction, further emphasizing the importance of plastic surgeons to remain at the forefront of these advocacy discussions. In contrast, another study analyzing cost-effectiveness of CPM compared to routine surveillance demonstrated favorable economic outcomes for CPM in average-risk women younger than 70 years of age (56). Cost effectiveness took into account quality of life differences between CPM and surveillance patients. CPM represents a surgical procedure warranting close evaluation with a focus on resource utilization given the controversy that surrounds its widespread application. In the current landscape of the US, healthcare quality is often equated to increased value with decreasing financial burden. Accordingly policymakers tend to advocate for improving overall healthcare by either decreasing cost and/or improving outcomes (57). This has led to an increasing emphasis on quality outcomes driving healthcare reimbursement models, thus encouraging a system to evolve to incentivizing better outcomes at a more affordable rate.

Although healthcare costs associated with CPM with reconstruction is most costly, the increased monetary cost of CPM may be offset by patient satisfaction and QOL (47). Moreover, in the current climate of healthcare utilization and move towards bundled payments, it will be imperative to justify the added healthcare costs associated with CPM by highlighting the improvements in patient satisfaction and well-being in order to ensure that this option remains a feasible option to all breast cancer patients who are making well informed decisions.

\section{Shared decision-making and patient education}

Shared decision-making is a collaborative process between a patient and their clinician to discuss options available to 
them using evidence-based, patient-centered data all while accounting for patient preferences (58). Plastic surgeons, although critical in these discussions, seem to take a more passive role in these discussions with patients about CPM, based on recent survey data (43). Given the complex considerations of CPM and subsequent breast reconstruction such as the impact of procedure type, increased risk of complications and potential for improvements in satisfaction and QOL, it is imperative that plastic surgeons take a more active role and engage in the shared decision-making process with patients considering CPM.

Understanding that patient education regarding overall breast cancer management and specifically CPM is relatively low, and that patient decisions about CPM are largely affected by knowledge of the issue (27), substantial opportunities for patient education exist. These opportunities are prime for clinicians and researchers not only to educate individual patients, but also the public, regarding breast cancer pathology, reconstructive options, and expected outcomes of CPM and breast reconstruction with the goal of optimizing the care we deliver to all patients faced with this complex decision.

\section{Acknowledgments}

Funding: None.

\section{Footnote}

Provenance and Peer Review: This article was commissioned by the Guest Editors (Charles E. Butler, Carrie Chu, and Margaret Roubaud) for the series "New Frontiers in Breast Reconstruction" published in Gland Surgery. The article was sent for external peer review organized by the Guest Editors and the editorial office.

Conflicts of Interest: All authors have completed the ICMJE uniform disclosure form (available at http://dx.doi. org/10.21037/gs.2020.03.15). The authors have no conflicts of interest to declare.

Ethical Statement: The authors are accountable for all aspects of the work in ensuring that questions related to the accuracy or integrity of any part of the work are appropriately investigated and resolved.

Open Access Statement: This is an Open Access article distributed in accordance with the Creative Commons
Attribution-NonCommercial-NoDerivs 4.0 International License (CC BY-NC-ND 4.0), which permits the noncommercial replication and distribution of the article with the strict proviso that no changes or edits are made and the original work is properly cited (including links to both the formal publication through the relevant DOI and the license). See: https://creativecommons.org/licenses/by-nc-nd/4.0/.

\section{References}

1. Panchal H, Pilewskie ML, Sheckter CC, et al. National trends in contralateral prophylactic mastectomy in women with locally advanced breast cancer. J Surg Oncol 2019;119:79-87.

2. Sabel MS, Kraft CT, Griffith KA, et al. Differences between Breast Conservation-Eligible Patients and Unilateral Mastectomy Patients in Choosing Contralateral Prophylactic Mastectomies. Breast J 2016;22:607-15.

3. Reiner AS, Sisti J, John EM, et al. Breast Cancer Family History and Contralateral Breast Cancer Risk in Young Women: An Update From the Women's Environmental Cancer and Radiation Epidemiology Study. J Clin Oncol 2018;36:1513-20.

4. Wong SM, Freedman RA, Sagara Y, et al. Growing Use of Contralateral Prophylactic Mastectomy Despite no Improvement in Long-term Survival for Invasive Breast Cancer. Ann Surg 2017;265:581-9.

5. Murthy V, Chamberlain RS. Prophylactic mastectomy in patients at high risk: is there a role for sentinel lymph node biopsy? Clin Breast Cancer 2013;13:180-7.

6. Nasser SM, Smith SG, Chagpar AB. The role of sentinel node biopsy in women undergoing prophylactic mastectomy. J Surg Res 2010;164:188-92.

7. Boughey JC, Attai DJ, Chen SL, et al. Contralateral Prophylactic Mastectomy (CPM) Consensus Statement from the American Society of Breast Surgeons: Data on CPM Outcomes and Risks. Ann Surg Oncol 2016;23:3100-5.

8. Bedrosian I, Hu CY, Chang GJ. Population-based study of contralateral prophylactic mastectomy and survival outcomes of breast cancer patients. J Natl Cancer Inst 2010;102:401-9.

9. Herrinton LJ, Barlow WE, Yu O, et al. Efficacy of prophylactic mastectomy in women with unilateral breast cancer: a cancer research network project. J Clin Oncol 2005;23:4275-86.

10. Boughey JC, Hoskin TL, Degnim AC, et al. Contralateral prophylactic mastectomy is associated with a survival 
advantage in high-risk women with a personal history of breast cancer. Ann Surg Oncol 2010;17:2702-9.

11. Carbine NE, Lostumbo L, Wallace J, et al. Risk-reducing mastectomy for the prevention of primary breast cancer. Cochrane Database Syst Rev 2018;4:CD002748.

12. Albornoz CR, Matros E, Lee CN, et al. Bilateral Mastectomy versus Breast-Conserving Surgery for EarlyStage Breast Cancer: The Role of Breast Reconstruction. Plast Reconstr Surg 2015;135:1518-26.

13. Buchanan PJ, Abdulghani M, Waljee JF, et al. An Analysis of the Decisions Made for Contralateral Prophylactic Mastectomy and Breast Reconstruction. Plast Reconstr Surg 2016;138:29-40.

14. Sando IC, Billig JI, Ambani SW, et al. An Evaluation of the Choice for Contralateral Prophylactic Mastectomy and Patient Concerns About Recurrence in a Reconstructed Cohort. Ann Plast Surg 2018;80:333-8.

15. Jagsi R, Hawley ST, Griffith KA, et al. Contralateral Prophylactic Mastectomy Decisions in a Population-Based Sample of Patients With Early-Stage Breast Cancer. JAMA Surg 2017;152:274-82.

16. Metcalfe KA, Lubinski J, Ghadirian P, et al. Predictors of contralateral prophylactic mastectomy in women with a BRCA1 or BRCA2 mutation: the Hereditary Breast Cancer Clinical Study Group. J Clin Oncol 2008;26:1093-7.

17. Rebbeck TR, Friebel T, Lynch HT, et al. Bilateral prophylactic mastectomy reduces breast cancer risk in BRCA1 and BRCA2 mutation carriers: the PROSE Study Group. J Clin Oncol 2004;22:1055-62.

18. Bevers TB, Armstrong DK, Arun B, et al. Breast cancer risk reduction. J Natl Compr Canc Netw 2010;8:1112-46.

19. King TA, Sakr R, Patil S, et al. Clinical management factors contribute to the decision for contralateral prophylactic mastectomy. J Clin Oncol 2011;29:2158-64.

20. Katipamula R, Degnim AC, Hoskin T, et al. Trends in mastectomy rates at the Mayo Clinic Rochester: effect of surgical year and preoperative magnetic resonance imaging. J Clin Oncol 2009;27:4082-8.

21. Miller BT, Abbott AM, Tuttle TM. The influence of preoperative MRI on breast cancer treatment. Ann Surg Oncol 2012;19:536-40.

22. Sorbero ME, Dick AW, Beckjord EB, et al. Diagnostic breast magnetic resonance imaging and contralateral prophylactic mastectomy. Ann Surg Oncol 2009;16:1597-605.

23. Hawley ST, Jagsi R, Morrow M, et al. Social and Clinical Determinants of Contralateral Prophylactic Mastectomy.
JAMA Surg 2014;149:582-9.

24. Lehman CD, Gatsonis C, Kuhl CK, et al. MRI evaluation of the contralateral breast in women with recently diagnosed breast cancer. $\mathrm{N}$ Engl J Med 2007;356:1295-303.

25. Boetes C, Mus RD, Holland R, et al. Breast tumors: comparative accuracy of MR imaging relative to mammography and US for demonstrating extent. Radiology 1995;197:743-7.

26. Houssami N, Hayes DF. Review of preoperative magnetic resonance imaging (MRI) in breast cancer: should MRI be performed on all women with newly diagnosed, early stage breast cancer? CA Cancer J Clin 2009;59:290-302.

27. Hooper RC, Hsu J, Duncan A, et al. Breast Cancer Knowledge and Decisions Made for Contralateral Prophylactic Mastectomy: A Survey of Surgeons and Women in the General Population. Plast Reconstr Surg 2019;143:936e-45e.

28. Rosenberg SM, Tracy MS, Meyer ME, et al. Perceptions, knowledge, and satisfaction with contralateral prophylactic mastectomy among young women with breast cancer: a cross-sectional survey. Ann Intern Med 2013;159:373-81.

29. Parker PA, Peterson SK, Bedrosian I, et al. Prospective Study of Surgical Decision-making Processes for Contralateral Prophylactic Mastectomy in Women With Breast Cancer. Ann Surg 2016;263:178-83.

30. Parker PA, Peterson SK, Shen Y, et al. Prospective Study of Psychosocial Outcomes of Having Contralateral Prophylactic Mastectomy Among Women With Nonhereditary Breast Cancer. J Clin Oncol 2018;36:2630-8.

31. Frost MH, Hoskin TL, Hartmann LC, et al. Contralateral prophylactic mastectomy: long-term consistency of satisfaction and adverse effects and the significance of informed decision-making, quality of life, and personality traits. Ann Surg Oncol 2011;18:3110-6.

32. Yi M, Hunt KK, Arun BK, et al. Factors affecting the decision of breast cancer patients to undergo contralateral prophylactic mastectomy. Cancer Prev Res (Phila) 2010;3:1026-34.

33. Orom H, Kiviniemi MT, Shavers VL, et al. Perceived risk for breast cancer and its relationship to mammography in Blacks, Hispanics, and Whites. J Behav Med 2013;36:466-76.

34. Davids SL, Schapira MM, McAuliffe TL, et al. Predictors of pessimistic breast cancer risk perceptions in a primary care population. J Gen Intern Med 2004;19:310-5.

35. Momoh AO, Cohen WA, Kidwell KM, et al. Tradeoffs 
Associated With Contralateral Prophylactic Mastectomy in Women Choosing Breast Reconstruction: Results of a Prospective Multicenter Cohort. Ann Surg 2017;266:158-64.

36. Hawley ST, Griggs JJ, Hamilton AS, et al. Decision involvement and receipt of mastectomy among racially and ethnically diverse breast cancer patients. J Natl Cancer Inst 2009; 101:1337-47.

37. Cooper LA, Beach MC, Johnson RL, et al. Delving below the surface. Understanding how race and ethnicity influence relationships in health care. J Gen Intern Med 2006;21 Suppl 1:S21-7.

38. Blair IV, Steiner JF, Fairclough DL, et al. Clinicians' implicit ethnic/racial bias and perceptions of care among Black and Latino patients. Ann Fam Med 2013;11:43-52.

39. Momoh AO, Griffith KA, Hawley ST, et al. Patterns and Correlates of Knowledge, Communication, and Receipt of Breast Reconstruction in a Modern Population-Based Cohort of Patients with Breast Cancer. Plast Reconstr Surg 2019;144:303-13.

40. Katz SJ, Hawley ST, Hamilton AS, et al. Surgeon Influence on Variation in Receipt of Contralateral Prophylactic Mastectomy for Women With Breast Cancer. JAMA Surg 2018;153:29-36.

41. Covelli AM, Baxter NN, Fitch MI, et al. Increasing mastectomy rates-the effect of environmental factors on the choice for mastectomy: a comparative analysis between Canada and the United States. Ann Surg Oncol 2014;21:3173-84.

42. Dobke MK, Yee B, Mackert GA, et al. The Influence of Patient Exposure to Breast Reconstruction Approaches and Education on Patient Choices in Breast Cancer Treatment. Ann Plast Surg 2019;83:206-10.

43. Momoh AO, Griffith KA, Hawley ST, et al. PostMastectomy Breast Reconstruction: Exploring Plastic Surgeon Practice Patterns and Perspectives. Plast Reconstr Surg 2020;145:865-76.

44. Silva AK, Lapin B, Yao KA, et al. The Effect of Contralateral Prophylactic Mastectomy on Perioperative Complications in Women Undergoing Immediate Breast Reconstruction: A NSQIP Analysis. Ann Surg Oncol 2015;22:3474-80.

45. Boughey JC, Hoskin TL, Hartmann LC, et al. Impact of reconstruction and reoperation on long-term patientreported satisfaction after contralateral prophylactic mastectomy. Ann Surg Oncol 2015;22:401-8.

46. Miller ME, Czechura T, Martz B, et al. Operative risks associated with contralateral prophylactic mastectomy: a single institution experience. Ann Surg Oncol
2013;20:4113-20.

47. Billig JI, Duncan A, Zhong L, et al. The Cost of Contralateral Prophylactic Mastectomy in Women with Unilateral Breast Cancer. Plast Reconstr Surg 2018;141:1094-102.

48. Alba B, Schultz BD, Cohen D, et al. Risk-to-Benefit Relationship of Contralateral Prophylactic Mastectomy: The Argument for Bilateral Mastectomies with Immediate Reconstruction. Plast Reconstr Surg 2019;144:1-9.

49. Sinha I, Pusic AL, Wilkins EG, et al. Late SurgicalSite Infection in Immediate Implant-Based Breast Reconstruction. Plast Reconstr Surg 2017;139:20-8.

50. Bennett KG, Qi J, Kim HM, et al. Comparison of 2-Year Complication Rates Among Common Techniques for Postmastectomy Breast Reconstruction. JAMA Surg 2018;153:901-8.

51. Santosa KB, Qi J, Kim HM, et al. Long-term PatientReported Outcomes in Postmastectomy Breast Reconstruction. JAMA Surg 2018;153:891-9.

52. Hwang ES, Locklear TD, Rushing CN, et al. PatientReported Outcomes After Choice for Contralateral Prophylactic Mastectomy. J Clin Oncol 2016;34:1518-27.

53. Craft RO, Colakoglu S, Curtis MS, et al. Patient satisfaction in unilateral and bilateral breast reconstruction [outcomes article]. Plast Reconstr Surg 2011;127:1417-24.

54. Deshmukh AA, Cantor SB, Crosby MA, et al. Cost of contralateral prophylactic mastectomy. Ann Surg Oncol 2014;21:2823-30.

55. Keskey RC, LaJoie AS, Sutton BS, et al. Cost-effectiveness Analysis of Contralateral Prophylactic Mastectomy Compared to Unilateral Mastectomy with Routine Surveillance for Unilateral, Sporadic Breast Cancer. Ann Surg Oncol 2017;24:3903-10.

56. Zendejas B, Moriarty JP, O'Byrne J, et al. Costeffectiveness of contralateral prophylactic mastectomy versus routine surveillance in patients with unilateral breast cancer. J Clin Oncol 2011;29:2993-3000.

57. Atkins JH, Fleisher LA. Value from the Patients' and Payers' Perspectives. Anesthesiol Clin 2015;33:651-8.

58. Politi MC, Lee CN, Philpott-Streiff SE, et al. A Randomized Controlled Trial Evaluating the BREASTChoice Tool for Personalized Decision Support About Breast Reconstruction After Mastectomy. Ann Surg 2020;271:230-7.

Cite this article as: Santosa KB, Oliver JD, Momoh AO. Contralateral prophylactic mastectomy and implications for breast reconstruction. Gland Surg 2021;10(1):498-506. doi: 10.21037 /gs.2020.03.15 